\title{
CORRECTION
}

\section{THE ROLE OF THE DRIFT IN I(2) SYSTEMS}

\author{
P. Paruolo \\ Department of Statistics, University of Bologna, 40126 Bologna, Italy \\ B. Nielsen \\ Nuffield College, Oxford, OX1 INF, United Kingdom
}

J. It. Statist. Soc. (1994) 3, pp. 93-123

\section{Summary}

A correction of the proof of Theorem 5.1 is given. All the statements of the Theorem and the Tables associated with it are unaffected by the correction.

In the Appendix, bottom of page 111, the term $\beta_{I}^{\prime} C_{I} W_{u}$ in the definition of $F_{u}^{*}$ should be replaced by $\left(l, \gamma_{1}\right)^{\prime} W_{u}$, where $l \equiv \bar{\tau}_{1}^{\prime} C_{l}-\frac{I}{2} \bar{\tau}_{2}{ }_{2} C_{2}$ and $\beta$ is chosen as $\beta_{l} \equiv\left(\bar{\tau}_{l}, \gamma_{l}\right)$, see Paruolo (1998), lemma A3. Let $B_{2 l u} \equiv c_{l}^{-1 / 2} V_{\alpha_{z^{4}}}=$ $c_{I}^{-1 / 2} f^{\prime} \alpha^{\prime}{ }_{2} W_{u}$, where $f^{\prime} \equiv \gamma_{2}^{\prime} \beta_{2}\left(\alpha_{2}^{\prime} \theta \beta_{2}\right)^{-1}, c_{1} \equiv f^{\prime} \alpha_{2}^{\prime} \Omega \alpha_{2} f$. Note that the process $V_{\alpha_{2} u}$ can be substituted with $B_{2 / u}$ in the definition of $F_{u}^{*}$, since $V_{\alpha_{2} u}$ is a bijective linear function of $B_{2 / u}$. Define also $B_{22 u} \equiv c_{2}^{-1 / 2} \bar{f}_{1}^{\prime}\left(\alpha_{2}^{\prime} \Omega \alpha_{2}\right)^{-1} \alpha_{2}^{\prime} W_{u}, c_{2} \equiv$ $\bar{f}_{\perp}^{\prime}\left(\alpha_{2}^{\prime} \Omega \alpha_{2}\right)^{-1} \bar{f}_{\perp} ; B_{21}$ and $B_{22}$ are independent Brownian standard motion of dimensions $p_{2}-l$ and $l$ respectively, and $B_{2}$ can be chosen equal to $\left(B_{2 l}^{\prime}, B_{22}\right)^{\prime}, B_{2 u}$ $=\left(B_{2 / \omega}^{\prime} B_{22 u}\right)^{\prime}$, where $B_{l}$ and $B_{2}$ are defined before (A.22). Next note that $\left(l, \gamma_{l}\right)^{\prime} W_{u}$ is a linear function of $\left(B^{\prime}{ }_{1}, B_{2}^{\prime}\right)^{\prime}$, so that $\left(l, \gamma_{l}\right)^{\prime} W_{u}=m_{l} B_{l u}+m 2_{l} B_{2 / u}+n B_{22 u}$, for appropriate matrices $m_{l,}, m_{2 l}, n$; hence $\left(\left(l, \gamma_{l}\right)^{\prime} W_{u} \mid 1, u, B_{2 l u}\right)=\left(m_{l} B_{l u}+n B_{22 u} \mid 1, G_{u}\right)$.

Define next $g_{I} \equiv\left(n_{\perp} c_{3}^{-1 / 2}, n c_{4}^{-1 / 2}\right), c_{3} \equiv n_{\perp}^{\prime} m_{l} m_{1}^{\prime} n_{\perp}, c_{4} \equiv c_{5}+\left(n^{\prime} n\right)^{2}, c_{5} \equiv$ $n^{\prime} m_{l} m^{\prime}{ }_{1} n$ and let $B_{I / u} \equiv c_{3}^{-1 / 2} n_{\perp}^{\prime} m_{l} B_{l u}, B_{l 2 u} \equiv c_{5}^{-1 / 2} n^{\prime} m_{l} B_{l u}$; one thus has

$$
\left(g_{1}^{\prime}\left(l, \gamma_{1}\right)^{\prime} W_{u} \mid 1, G_{u}\right)=\left(\left(B_{11 u}^{\prime}, k_{l} B_{12 u}+k_{2} B_{22 u}\right)^{\prime} \mid 1, G_{u}\right)
$$

where $k_{1} \equiv\left(c_{5} / c_{4}\right)^{-1 / 2}, k_{2}^{2}=1-k_{1}^{2}$ and $k_{1} B_{l 2 u}+k_{2} B_{22 u}$ is a standard univariate Brownian motion independent of $B_{\| l}$ and $B_{2 l}$. 
The proof of Theorem 5.1 on page 115, between eq. (A.22) and (A.23) should be changed as follows. Let $g \equiv \operatorname{diag}\left(g_{l}, c_{l}^{-1 / 2}, 1\right)$, and define $F \equiv g^{\prime} F_{u}^{*}=$ $\left\{\left(B_{11 u}^{\prime}, k_{l} B_{12 u}+k_{2} B_{22 u}, U_{2 l u}^{\prime}, u^{2}\right)^{\prime} \mid 1, u, B_{2 I u}\right\}$; pre-multiplying (A.22) by $g^{\prime}$ and post-multiplying it by $g$ one obtains

$$
{ }_{2} Q\left(p_{0}\right) \rightarrow^{w} \sum_{i=p_{0}+1}^{p} \psi_{i}=h(B, F)
$$

where $h\left(x_{u}, y_{u}\right) \equiv \operatorname{tr}\left\{\int_{0}^{l}(d x) y^{\prime}\left(\int_{0}^{l} y y^{\prime} d u\right)^{-1} \int_{0}^{l} y(d x)^{\prime}\right\}$

The rest of the proof is unaltered; thus ${ }_{2} Q\left(p_{1} \mid p_{0}\right) \rightarrow{ }^{w} h\left(B_{2}, G\right)$ and ${ }_{2} Q\left(p_{0}, p_{l}\right)$ $\rightarrow^{w} h(B, F)+h\left(B_{2}, G\right)$. We want to show that the distribution of $h(B, F)+h\left(B_{2}\right.$, $G$ ) does not depend on $k_{l}$, and hence on $k_{2}$. The statement of Theorem 5.1 then follows by choosing $k_{l}=1-k_{2}=1$.

We start by showing that the two terms of $h(B, F)+h\left(B_{2}, G\right)$ are conditionally independent given $g \equiv \sigma\left(B_{1 l u}, B_{2 I u}, u \in[0, I]\right)$. We then proceed to show that the conditional distribution of the first term, $h(B, F)$, and hence of $h(B, F)+h\left(B_{2}, G\right)$ does not depend on $k_{l}$. Consequently also the unconditional distribution does not depend on $k_{l}$.

In the following the information set $\mathscr{g}$ is held fixed. The only random element of the first term, $h(B, F)$, is $J \equiv \int_{0}^{l} F d\left(B_{12}^{\prime} B_{22}\right)$ whereas the only random element of the second term $h\left(B_{2}, G\right)$, is $K \equiv \int_{0}^{l} G d B_{22}$. When conditioning on $\mathscr{g}$ the process $G_{u}$ is fixed and, except for the component $k_{l} B_{l 2}+k_{2} B_{22}$, the process $F_{u}$ is fixed as well. The two terms have the process $B_{22}$ in common. It will be argued that $J$ depends only on $B_{22}$ through the process

$$
B_{22 u}^{o} \equiv B_{22 u}-\int_{0}^{u} G_{s}^{\prime} d s\left(\int_{0}^{l} G_{s} G_{s}^{\prime} d s\right)^{-1} \int_{0}^{I} G_{s} d B_{22}=B_{22 u}-\int_{0}^{u} G_{s}^{\prime} d s \nVdash
$$

The process $B_{22 u}^{o}$ and the variable $K$ are both Gaussian and arise from the same process, $B_{22}$. Consequently, $B_{22 u}^{o}$ and $K$, and hence also $J$ and $K$, are independent since their cross variation is zero

$$
\int_{0}^{l}\left\{l_{(t \leq u)}-\int_{0}^{u} G_{s}^{\prime} d s\left(\int_{0}^{I} G_{s} G_{s}^{\prime} d s\right)^{-I} G_{t}\right\} G_{t}^{\prime} d t=0
$$


The process $B_{22}$ occurs both in the integrator and the integrand of the integral $J \equiv \int_{0}^{l} F d\left(B_{12}^{\prime}, B_{22}\right)$. First, it is argued that $\int_{0}^{I} F d B_{22}=\int_{0}^{l} F d B_{22}^{o}$. Use of $(\mathrm{C} .1)$ gives

$$
\int_{0}^{l} F d B_{22}=\int_{0}^{l} F d B_{22}^{o}+\int_{0}^{I} F G^{\prime} d s \not
$$

Since $F$ is corrected for $G$ the integral $\int_{0}^{l} F G^{\prime} d s=0$. Secondly, divide the process $F$ into two components: $F_{l}=\left(B_{l 1 u}, k_{l} B_{12 u}+k_{2} B_{22 u}\right)$ and $F_{2}=\int_{0}^{u} G_{s} d s$ which is fixed given $\mathcal{G}$. The $h$-function has the property that $h(B, F)=h(B, M F)$ for any non-singular matrix $M$. Thus, $F$ can be replaced by the vector of $F_{1} \mid F_{2}$ and $F_{2}$. Using (C.1) once again it follows that

$$
\begin{aligned}
\left(F_{l} \mid F_{2}\right) & =\left(\left(B_{11 u}^{\prime} k_{l} B_{I 2 u}+k_{2} B_{22 u}\right)^{\prime} \mid 1, G, \int_{0}^{u} G\right)= \\
& =\left(\left(B_{I I u}^{\prime} k_{l} B_{12 u}+k_{2} B_{22 u}^{o}\right)^{\prime} \mid 1, G, \int_{0}^{u} G\right)
\end{aligned}
$$

Consequently, $h(B, F)$ depends on $B_{22}$ only through $B_{22}^{o}$, and hence $h(B, F)$ and $h\left(B_{2}, G\right)$ are conditionally independent for fixed $\mathscr{G}$.

Next, it has to be shown that the conditional distribution of the first term, $h(B$, $F)$, does not depend on $k_{1}$. By standard properties of the trace, the $h$-function has the property that $h(B, F)=h(L B, F)$ for any orthogonal matrix $L$. Define

$$
L=\left(\begin{array}{cccc}
I_{p_{1}-1} & 0 & 0 & 0 \\
0 & k_{1} & 0 & k_{2} \\
0 & 0 & I_{p_{2}-1} & 0 \\
0 & k_{2} & 0 & -k_{1}
\end{array}\right) \quad L^{-I}=L^{\prime}
$$

and the process $B_{u}^{+}=L B_{u}$ which is a standard Brownian motion. Since $F^{\prime}=$ $\left(B_{11}^{\prime}, B_{12}^{+}, \int_{0}^{u} G^{\prime} \mid 1, G\right), h(B, F)$ can be expressed as $h\left(B^{+}, F\right)$ in terms of a Brownian motion $B^{+}$not involving $k_{l}$.

\section{REFERENCE}

Paruolo P. (1998), Asymptotic efficiency of the two stage estimator in I(2) systems, forthcoming in Econometric Theory. 